\title{
An idea which yields a lot of elementary inequalities
}

\author{
ISTVÁN SZALAY
}

Abstract. The aim of the article is to show how studies in higher mathematics can be applied in everyday teaching practice to construct new problems for their pupils. In higher mathematics it is known that the set of real numbers with the addition and multiplication (shortly: $(\mathbb{R},+, \times))$ is an ordered field. Considering a strictly monotonic increasing and continuous function $\sigma$ with domain $D_{\sigma}=\mathbb{R}=\{x:-\infty<x<\infty\}$ and range $\mathbb{R}_{\sigma}=\{\xi \in \mathbb{R}:-1<\xi<1\}$, the map $x \rightarrow \sigma(x)$ is a bijection between $\mathbb{R}$ and the open interval $(-1,1)$. Introducing the operations sub-addition $\xi \oplus \eta=\sigma\left(\sigma^{-1}(\xi)+\sigma^{-1}(\eta)\right)$ and sub-multiplication $\xi \otimes \eta=\sigma\left(\sigma^{-1}(\xi) \times \sigma^{-1}(\eta)\right)$ we have the ordered field $\left(\mathbb{R}_{\sigma}, \oplus, \otimes\right)$, where for any pair $\xi=\sigma(x), \eta=\sigma(y) ; x, y \in \mathbb{R}$ the ordering in $\mathbb{R}_{\sigma}$ means that $\xi<\eta$ if and only if $x<y$. Consequently, if $\xi, \eta \in(-1,1)$ then $-1<\xi \oplus \eta<1 ;-1<\xi \otimes \eta<1$. By this idea, using different kinds of functions $\sigma$ we show a lot of different elementary inequalities.

Key words and phrases: elementary inequality.

ZDM Subject Classification: H40.

1. The $\sigma$ functions and their operations

Trying to find some suitable functions we have that the functions

$$
\begin{aligned}
\sigma_{H}(x) & =\operatorname{th} x=\frac{e^{x}-e^{-x}}{e^{x}+e^{-x}} \\
\sigma_{T}(x) & =\frac{2}{\pi} \operatorname{arctg} \frac{\pi \cdot x}{2}
\end{aligned}
$$

Supported by Foundation Domonkos Bonifert, Szeged, Hungary.

Copyright (C) 2010 by University of Debrecen 
and

$$
\sigma_{G}(x)=\frac{x}{1+|x|}
$$

have the set of real numbers $\mathbb{R}$ as their domain and the open interval $(-1,1)$ as their range. For the sake of simplicity we write

$$
\sigma_{H}(x)=\underline{x}_{H}, \quad \sigma_{T}(x)=\underline{x}_{T}, \quad \sigma_{G}(x)=\underline{x}_{G}, \quad x \in \mathbb{R}
$$

and

$$
\begin{array}{cc}
\sigma_{H}^{-1}(\xi)=\operatorname{areath} \xi=\frac{1}{2} \ln \frac{1+\xi}{1-\xi}=\bar{\xi}_{H}, & \sigma_{T}^{-1}(\xi)=\frac{2}{\pi} \operatorname{tg} \frac{\pi \cdot \xi}{2}=\bar{\xi}_{T}, \\
\sigma_{G}^{-1}(\xi)=\frac{\xi}{1-|\xi|}=\bar{\xi}_{G}, \quad \xi \in(-1,1) .
\end{array}
$$

Moreover,

$$
\mathbb{R}_{\sigma_{H}}=\mathbb{R}_{\sigma_{T}}=\mathbb{R}_{\sigma_{G}}=(-1,1)=\underline{\mathbb{R}}
$$

and we write that

$$
\underline{\mathbb{R}^{+}}=\{\xi \in \mathbb{R}: 0 \leq \xi<1\}=[0,1) .
$$

So,

$$
\begin{gathered}
\xi \oplus_{H} \eta=\bar{\xi}_{H}+\bar{\eta}_{H}, \quad \xi \otimes_{H} \eta=\underline{\bar{\xi}_{H} \times \bar{\eta}_{H}}, \quad \xi \oplus_{T} \eta=\underline{\bar{\xi}_{T}+\bar{\eta}_{T}}, \\
\xi \otimes_{T} \eta=\underline{\bar{\xi}_{T} \times \bar{\eta}_{T}}, \quad \xi \oplus_{G} \eta=\underline{\bar{\xi}_{G}+\bar{\eta}_{G}} \text { and } \xi \otimes_{G} \eta=\underline{\bar{\xi}_{G} \times \bar{\eta}_{G}} .
\end{gathered}
$$

Hence, we get that for any pair $\xi, \eta \in \underline{\mathbb{R}}$

$$
\begin{aligned}
& \xi \oplus_{H} \eta=\operatorname{th}(\operatorname{areath} \xi+\operatorname{areath} \eta)=\frac{\xi+\eta}{1+\xi \cdot \eta} \in \underline{\mathbb{R}} \\
& \xi \otimes_{H} \eta=\operatorname{th}(\operatorname{areath} \xi \cdot \operatorname{areath} \eta) \in \underline{\mathbb{R}} \\
& \xi \oplus_{T} \eta=\frac{2}{\pi} \operatorname{arctg}\left(\operatorname{tg} \frac{\pi \cdot \xi}{2}+\operatorname{tg} \frac{\pi \cdot \eta}{2}\right) \in \underline{\mathbb{R}} \\
& \xi \otimes_{T} \eta=\frac{2}{\pi} \operatorname{arctg}\left(\frac{2}{\pi}\left(\operatorname{tg} \frac{\pi \cdot \xi}{2}\right) \cdot\left(\operatorname{tg} \frac{\pi \cdot \eta}{2}\right)\right) \in \underline{\mathbb{R}} \\
& \xi \oplus_{G} \eta=\frac{\xi+\eta-\xi|\eta|-\eta|\xi|}{1-|\xi|-|\eta|+|\xi \eta|+|\xi+\eta-\xi| \eta|-\eta| \xi||} \in \underline{\mathbb{R}}
\end{aligned}
$$

and

$$
\xi \otimes_{G} \eta=\frac{\xi \eta}{1-|\xi|-|\eta|+2|\xi \eta|} \in \underline{\mathbb{R}}
$$




$$
\text { "tmcs-szalay" — 2010/4/12 — 23:36 — page } 63 \text { — \#3 }
$$

hold. Sub-addition under (1.4) is very comfortable, so we will use the Abel group $\left(\underline{\mathbb{R}}, \oplus_{H}\right) . \quad\left(\underline{\mathbb{R}}, \oplus_{H}, \otimes_{H}\right)$ is an ordered field but sub-multiplication under (1.5) is not comfortable enough, so we do not investigate it. Sub-addition under (1.6) and sub-multiplication under (1.7) are uncomfortable, so we can see that the $\sigma$ function under (1.2) is not suitable for finding elementary inequalities.

Having that if $\xi, \eta \in \underline{\mathbb{R}}$ then $|\xi \eta|<1$, we consider the multiplication

$$
\xi \otimes \eta=\xi \eta \in \underline{\mathbb{R}}
$$

too. (We remark that $\left(\underline{\mathbb{R}}, \oplus_{H}, \otimes\right)$ is not a field because the distributivity

$$
\xi \otimes\left(\eta \oplus_{H} \zeta\right)=(\xi \otimes \eta) \oplus_{H}(\xi \otimes \zeta), \quad \xi, \eta, \zeta \notin \underline{\mathbb{R}}
$$

is not valid except the cases $\xi=0$ or $\eta=-\zeta$.) Anyhow, $\left(\underline{\mathbb{R}}, \oplus_{H}, \otimes\right)$ is a closed algebraic structure. Sub-addition under (1.8) and sub-multiplication under (1.9) become comfortable with the assumption $\xi, \eta \in \underline{\mathbb{R}^{+}}$, because (1.8) and (1.9) are reduced

and

$$
\xi \oplus_{G} \eta=\frac{\xi+\eta-2 \xi \eta}{1-\xi \eta} \in \underline{\mathbb{R}^{+}}
$$

$$
\xi \otimes_{G} \eta=\frac{\xi \eta}{1-\xi-\eta+2 \xi \eta} \in \underline{\mathbb{R}^{+}},
$$

respectively. As the functions under (1.1) and (1.3) give isomorhisms between $\mathbb{R}^{+}=\{x \in \mathbb{R}: 0 \leq x<\infty\}$ and $\underline{\mathbb{R}}$, we have that $\left(\underline{\mathbb{R}^{+}}, \oplus_{H}, \oplus_{G}, \otimes_{G}, \otimes\right)$ is a closed algebraic structure.

\section{Basic inequalities}

The definitions and results, mentioned in Section 1 are understandable for teachers or students, but not for pupils. For the sake of pupils we use $\xi=a$, $\eta=b \ldots$ and so on. The Problem 1 below based on (1.4).

Problem 1. Is it true that if $-1<a, b<1$ then $-1<\frac{a+b}{1+a b}<1$ ?

Problem 1 is already understandable for pupils, too. Of course, they have to give an elementary solution. For example: As $0<1+a b$ the original inequality is equivalent to $-1-a b<a+b<1+a b$. Now the inequality on the left is true, because $-(1+a)(1+b)<0$ and the inequality on the right is true because $0<(1-a)(1-b)$. 


$$
\text { "tmcs-szalay" — 2010/4/12 — 23:36 — page } 64 \text { — \#4 }
$$

István Szalay

Problem 2 (Based on 1.11). Is it true that if $0 \leq a, b<1$ then

$$
0 \leq \frac{a+b-2 a b}{1-a b}<1 ?
$$

An elementary solution: As $0<1-a b$ the original inequality is equivalent to $0 \leq a+b-2 a b<1-a b$. For "left" inequality we use $\frac{a+b}{2} \geq \sqrt{a b} \geq a b$ while the "right" we use $a+b<1+a b$, which has already been prove in the solution of Problem 1.

Problems 1 and 2 induce

Problem 2.1. Let $a$ and $b$ be real numbers. Let us solve the inequality $a+b<1+a b$.

(Solution: By $0<(1-a)(1-b)$ we have that $a+b<1+a b$ if and only if $(-\infty<a, b<1)$ or $(1<a, b<\infty)$.)

Problem 3 (Based on (1.12)). Is it true that if $0 \leq a, b<1$ then

$$
0 \leq \frac{a b}{1-a-b+2 a b}<1 ?
$$

First of all, the question is

Problem 3.1. Is it true that if $0 \leq a, b<1$ then $a+b<1+2 a b$ ?

By the solution of Problem 2.1 the answer is "true".

Turning to the solution to Problem 3 , the inequality mentioned there is equivalent to $0 \leq a b<1-a-b+2 a b$. Clearly, the "left" inequality is true. For the "right" one we apply the solution to Problem 2.1, again.

\section{Inequalities with three variables}

Considering variables $a, b, c \in \underline{\mathbb{R}}$ or $\underline{\mathbb{R}^{+}}$and having operations $\oplus_{H}, \oplus_{G}, \otimes_{G}$, $\otimes$ we get the following 32 sources for the problems.

Source 1. If $a, b, c \in \underline{\mathbb{R}} \Rightarrow\left(a \oplus_{H} b\right) \oplus_{H} c \in \underline{\mathbb{R}}$.

Source 2. If $a, b, c \in \underline{\mathbb{R}} \Rightarrow a \oplus_{H}\left(b \oplus_{H} c\right) \in \underline{\mathbb{R}}$, see Source 1 .

Source 3. If $a, b, c \in \underline{\mathbb{R}^{+}} \Rightarrow\left(a \oplus_{H} b\right) \oplus_{G} c \in \underline{\mathbb{R}^{+}}$. 
Source 4. If $a, b, c \in \underline{\mathbb{R}^{+}} \Rightarrow a \oplus_{H}\left(b \oplus_{G} c\right) \in \underline{\mathbb{R}^{+}}$.

Source 5. If $a, b, c \in \underline{\mathbb{R}^{+}} \Rightarrow\left(a \oplus_{H} b\right) \otimes_{G} c \in \underline{\mathbb{R}^{+}}$.

Source 6. If $a, b, c \in \underline{\mathbb{R}^{+}} \Rightarrow a \oplus_{H}\left(b \otimes_{G} c\right) \in \underline{\mathbb{R}^{+}}$.

Source 7. If $a, b, c \in \underline{\mathbb{R}} \Rightarrow\left(a \oplus_{H} b\right) \otimes c \in \underline{\mathbb{R}}$.

Source 8. If $a, b, c \in \underline{\mathbb{R}} \Rightarrow a \oplus_{H}(b \otimes c) \in \underline{\mathbb{R}}$.

Source 9. If $a, b, c \in \underline{\mathbb{R}^{+}} \Rightarrow\left(a \oplus_{G} b\right) \oplus_{H} c \in \underline{\mathbb{R}^{+}}$, see Source 4.

Source 10. If $a, b, c \in \underline{\mathbb{R}^{+}} \Rightarrow a \oplus_{G}\left(b \oplus_{H} c\right) \in \underline{\mathbb{R}^{+}}$, see Source 3.

Source 11. If $a, b, c \in \underline{\mathbb{R}^{+}} \Rightarrow\left(a \oplus_{G} b\right) \oplus_{G} c \in \underline{\mathbb{R}^{+}}$.

Source 12. If $a, b, c \in \underline{\mathbb{R}^{+}} \Rightarrow a \oplus_{G}\left(b \otimes_{G} c\right) \in \underline{\mathbb{R}^{+}}$, see Source 11 .

Source 13. If $a, b, c \in \underline{\mathbb{R}^{+}} \Rightarrow\left(a \oplus_{G} b\right) \otimes_{G} c \in \underline{\mathbb{R}^{+}}$.

Source 14. If $a, b, c \in \underline{\mathbb{R}^{+}} \Rightarrow a \oplus_{G}\left(b \otimes_{G} c\right) \in \underline{\mathbb{R}^{+}}$.

Source 15. If $a, b, c \in \underline{\mathbb{R}^{+}} \Rightarrow\left(a \oplus_{G} b\right) \otimes c \in \underline{\mathbb{R}^{+}}$.

Source 16. If $a, b, c \in \underline{\mathbb{R}^{+}} \Rightarrow a \oplus_{G}(b \otimes c) \in \underline{\mathbb{R}^{+}}$,

Source 17. If $a, b, c \in \underline{\mathbb{R}^{+}} \Rightarrow\left(a \otimes_{G} b\right) \oplus_{H} c \in \underline{\mathbb{R}^{+}}$, see Source 6 .

Source 18. If $a, b, c \in \underline{\mathbb{R}^{+}} \Rightarrow a \otimes_{G}\left(b \oplus_{H} c\right) \in \underline{\mathbb{R}^{+}}$, see Source 5 .

Source 19. If $a, b, c \in \underline{\mathbb{R}^{+}} \Rightarrow\left(a \otimes_{G} b\right) \oplus_{G} c \in \underline{\mathbb{R}^{+}}$, see Source 14.

Source 20. If $a, b, c \in \underline{\mathbb{R}^{+}} \Rightarrow a \otimes_{G}\left(b \oplus_{G} c\right) \in \underline{\mathbb{R}^{+}}$, see Source 13.

Source 21. If $a, b, c \in \underline{\mathbb{R}^{+}} \Rightarrow\left(a \otimes_{G} b\right) \otimes_{G} c \in \underline{\mathbb{R}^{+}}$.

Source 22. If $a, b, c \in \underline{\mathbb{R}} \Rightarrow a \otimes_{G}\left(b \otimes_{G} c\right) \in \underline{\mathbb{R}^{+}}$, see Source 21 .

Source 23. If $a, b, c \in \underline{\mathbb{R}^{+}} \Rightarrow\left(a \otimes_{G} b\right) \otimes c \in \underline{\mathbb{R}^{+}}$.

Source 24. If $a, b, c \in \underline{\mathbb{R}} \Rightarrow a \otimes_{G}(b \otimes c) \in \underline{\mathbb{R}^{+}}$.

Source 25. If $a, b, c \in \underline{\mathbb{R}} \Rightarrow(a \otimes b) \oplus_{H} c \in \underline{\mathbb{R}}$, see Source 8 .

Source 26. If $a, b, c \in \mathbb{R} \Rightarrow a \otimes\left(b \oplus_{H} c\right) \in \underline{\mathbb{R}}$, see Source \%.

Source 2\%. If $a, b, c \in \underline{\mathbb{R}^{+}} \Rightarrow(a \otimes b) \oplus_{G} c \in \underline{\mathbb{R}^{+}}$, see Source 16 .

Source 28. If $a, b, c \in \underline{\mathbb{R}^{+}} \Rightarrow a \otimes\left(b \oplus_{G} c\right) \in \underline{\mathbb{R}^{+}}$, see Source 15.

Source 29. If $a, b, c \in \underline{\mathbb{R}^{+}} \Rightarrow(a \otimes b) \otimes_{G} c \in \underline{\mathbb{R}^{+}}$, see Source 24.

Source 30. If $a, b, c \in \underline{\mathbb{R}} \Rightarrow a \otimes\left(b \otimes_{G} c\right) \in \underline{\mathbb{R}^{+}}$, see Source 23.

Source 31. If $a, b, c \in \underline{\mathbb{R}} \Rightarrow(a \otimes b) \otimes c \in \mathbb{R}$. 
Source 32. If $a, b, c \in \underline{\mathbb{R}} \Rightarrow a \otimes(b \otimes c) \in \underline{\mathbb{R}}$, see Source 31 .

By the associativity and commutativity of operations we omit 16 sources signed by "see Source". Source 31, being trivial, is omitted, too. There remain Sources 1, 3-8, 11, 13-16, 21, 23 and 24. According to these sources we give the following 15 problems:

Problem 4 (Based on Source 1). Is it true that if $-1<a, b, c<1$ then

$$
-1<\frac{a+b+c+a b c}{1+a b+a c+b c}<1 ?
$$

Problem 5 (Based on Source 7). Is it true that if $-1<a, b, c<1$ then

$$
-1<\frac{a c+b c}{1+a b}<1 ?
$$

Problem 6 (Based on Source 8). Is it true that if $-1<a, b, c<1$ then

$$
-1<\frac{a+b c}{1+a b c}<1 ?
$$

Problem 7 (Based on Source 3). Is it true that if $0 \leq a, b, c<1$ then

$$
0 \leq \frac{a+b+c-2 a c-2 b c+a b c}{1+a b-a c-b c}<1 ?
$$

Problem 8 (Based on Source 4). Is it true that if $0 \leq a, b, c<1$ then

$$
0 \leq \frac{a+b+c-2 b c-a b c}{1+a b+a c-b c-2 a b c}<1 ?
$$

Problem 9 (Based on Source 5). Is it true that if $0 \leq a, b, c<1$ then

$$
0 \leq \frac{a c+b c}{1-a-b-c+a b+2 a c+2 b c-a b c}<1 ?
$$

Problem 10 (Based on Source 6). Is it true that if $0 \leq a, b, c<1$ then

$$
0 \leq \frac{a-a b-a c+b c+2 a b c}{1-b-c+2 b c+a b c}<1 ?
$$

Problem 11 (Based on Source 11). Is it true that if $0 \leq a, b, c<1$ then

$$
0 \leq \frac{a+b+c-2 a b-2 a c-2 b c+3 a b c}{1-a b-a c-b c+2 a b c}<1 ?
$$




$$
\text { "tmcs-szalay" — 2010/4/12 — 23:36 — page } 67 \text { — \#7 }
$$

Problem 12 (Based on Source 13). Is it true that if $0 \leq a, b, c<1$ then

$$
0 \leq \frac{a c+b c-2 a b c}{1-a-b-c+a b+2 a c+2 b c-3 a b c}<1 ?
$$

Problem 13 (Based on Source 14). Is it true that if $0 \leq a, b, c<1$ then

$$
0 \leq \frac{a-a b-a c}{1-b-c+2 b c-a b c}<1 ?
$$

Problem 14 (Based on Source 15). Is it true that if $0 \leq a, b, c,<1$ then

$$
0 \leq \frac{a c+b c-2 a b c}{1-a b}<1 ?
$$

Problem 15 (Based on Source 16). Is it true that if $0 \leq a, b, c<1$ then

$$
0 \leq \frac{a+b c-2 a b c}{1-a b c}<1 ?
$$

Problem 16 (Based on Source 21). Is it true that if $0 \leq a, b, c<1$ then

$$
0 \leq \frac{a b c}{1-a-b-c+a b+a c+b c}<1 ?
$$

Problem 17 (Based on Source 23). Is it true that if $0 \leq a, b, c<1$ then

$$
0 \leq \frac{a b c}{1-a-b+2 a b}<1 ?
$$

Problem 18 (Based on Source 24). Is it true that if $0 \leq a, b, c<1$ then

$$
0 \leq \frac{a b c}{1-a-b c+2 a b c}<1 ?
$$

According to sources we can see that in the cases of Problems 4-18 the answer is "yes". In the case of Problem 7, we show the method of constructing the problem by Source 3. The condition $a, b, c \in \underline{\mathbb{R}^{+}}$means that $0 \leq a, b, c<1$. Moreover, by (1.11) and (1.4) we get

$$
\begin{aligned}
\left(a \oplus_{H} b\right) \oplus_{G} c & =\frac{\left(a \oplus_{H} b\right)+c-2\left(a \oplus_{H} b\right) c}{1-\left(a \oplus_{H} b\right) c}=\frac{\frac{a+b}{1+a b}+c-2 \frac{a+b}{1+a b} c}{1-\frac{a+b}{1+a b} c} \\
& =\frac{a+b+c+a b c-2 a c-2 b c}{1+a b-a c-b c}
\end{aligned}
$$




$$
\text { "tmcs-szalay" — 2010/4/12 — 23:36 — page } 68 \text { — \#8 }
$$

and $\left(a \oplus_{H} b\right) \oplus_{G} c \in \underline{\mathbb{R}^{+}}$means that

$$
0 \leq \frac{a+b+c+a b c-2 a c-2 b c}{1+a b-a c-b c}<1 .
$$

This method is used for the other Problems, too. Of course, these solutions are not elementary solutions. Among elementary solutions there are difficult solutions and easy solutions, as well. But the judgements "difficult" or "easy" are relative. For example after Problem 1, the solution to Problem 5 is "easy" because if $\left|\frac{a+b}{1+a b}\right|<1$ and $|c|<1$, then $\left|\frac{(a+b) c}{1+a b}\right|<1$ is obvious. On the other hand, the elementary solution to Problem 4 is "difficult". First of all the following problem is raised:

Problem 4.1. Is it true that if $-1<a, b, c<1$ then $0<1+a b+a c+b c$ ?

Solution to Problem 4.1. By $0<1+a b$ we can write

$$
1+a b+a c+b c=(1+a b)\left(1+\frac{a c+b c}{1+a b}\right)
$$

so, considering that the answer to Problem 5 was "yes", we obtain that $0<1+a b+a c+b c$.

Turning to the solution to Problem 4, the inequality mentioned there is equivalent to inequality

$$
-1-a b-a c-b c<a+b+c+a b c<1+a b+a c+b c .
$$

Now the "left" inequality is true because $-(1+a)(1+b)(1+c)<0$ and the "right" inequality is true because $0<(1-a)(1-b)(1-c)$.

Each inequality with three variables is a source of inequalities with two variables. For example if in the case of Problem 4 writing $a=x, b=y, c=\frac{1}{2}$ we have

Problem 4.2. Is it true that if $-1<x, y<1$ then

$$
-1<\frac{x y+2 x+2 y+1}{2 x y+x+y+2}<1 ?
$$

We give two elementary solutions.

"Geometric" solution to Problem 4.2. Considering that the points $(x, y)$ where $2 x y+x+y+2=0$ are situated on a hyperbola we can see that 


$$
\text { "tmcs-szalay" — 2010/4/12 — 23:36 — page } 69 \text { — \#9 }
$$

"inner side" of the hyperbola $0<2 x y+x+y+2$ and the "outer side" of the hyperbola $2 x y+x+y+2<0$. Points $(x, y)$ with the condition $-1<x, y<1$ are the points of an open square "inside" the hyperbola, so $0<2 x y+x+y+2$. For the points of this open square inequality

$$
-1<\frac{x y+2 x+2 y+1}{2 x y+x+y+2}<1
$$

is equivalent to the inequality

$$
-2 x y-x-y-2<x y+2 x+2 y+1<2 x y+x+y+2 .
$$

Now the "left" inequality is true because $-3(x+1)(y+1)<0$ and the "right" inequality is true because $0<(x-1)(x+1)$. (We remark that this method is a way of the solution of inequality

$$
-1<\frac{x y+2 x+2 y+1}{2 x y+x+y+2}<1
$$

without any condition, too.)

"Algebraic" solution to Problem 4.2. By $0<1+x y$ we can write

$$
2 x y+x+y+2=2(1+x y)\left(1+\frac{1}{2} \frac{x+y}{1+x y}\right) .
$$

Considering that the answer to Problem 1 was "yes", we obtain that

$$
\frac{1}{2}<1+\frac{1}{2} \frac{x+y}{1+x y}<\frac{3}{2}
$$

so $0<2 x y+x+y+2$. Hence, the continuation is the same as in the "geometric" solution.

Finally we give the non-elementary solution to Problem 4.2: As $\frac{1}{2} \in \underline{\mathbb{R}}$, if $x, y \in \underline{\mathbb{R}}$, then

$$
x \oplus_{H} y \oplus_{H} \frac{1}{2}=\frac{2 x+2 y+1+x y}{2+2 x y+x+y} \in \underline{\mathbb{R}} . \quad \text { ( See Source 1.) }
$$

\section{Inequalities with four variables}

Considering variables $a, b, c, d \in \underline{\mathbb{R}}$ or $\underline{\mathbb{R}^{+}}$and having operations $\oplus_{H}, \oplus_{G}$, $\otimes_{G}, \otimes$ we get 320 sources for problems. Of course, by the associativity and 
commutativity of operations, certain sources are omitted but we have a lot of essentially different sources. For example

Source 33. If $a, b, c, d \in \underline{\mathbb{R}} \Rightarrow\left(a \oplus_{H} b\right) \oplus_{H}\left(c \oplus_{H} d\right) \in \underline{\mathbb{R}}$.

By this source we obtain

Problem 19. Is it true that if $-1<a, b, c, d<1$ then

$$
-1<\frac{a+b+c+d+a b c+a b d+a c d+b c d}{1+a b+a c+a d+b c+b d+c d+a b c d}<1 ?
$$

We can see that if $d=0$, Problem 19 gives back Problem 4. On the other hand, writing $c \oplus_{H} d \in \underline{\mathbb{R}}$ (see (1.4)) instead of $c$, and having that the answer to Problem 4 was "yes" by

$$
\frac{a+b+\left(c \oplus_{H} d\right)+a b\left(c \oplus_{H} d\right)}{1+a b+a\left(c \oplus_{H} d\right)+b\left(c \oplus_{H} d\right)}=\frac{a+b+c+\frac{c+d}{1+c d}+\frac{a b(c+d)}{1+c d}}{1+a b+\frac{a(c+d)}{1+c d}+\frac{b(c+d)}{1+c d}}
$$

we obtain that the answer to Problem 19 is "yes". This is a non - elementary solution. Turning an elementary solution to Problem 19, firstly we consider

Problem 19.1. Problem 4.1. Is it true that if $-1<a, b, c, d<1$ then $0<1+a b+a c+a d+b c+b d+c d+a b c d ?$

Solution to Problem 19.1. Considering that the answer for Problem 4.1 was "yes" we can write

$1+a b+a c+a d+b c+b d+c d+a b c d=(1+a b+a c+b c)\left(1+d \frac{a+b+c+a b c}{1+a b+a c+b c}\right)$,

moreover considering that the answer to Problem 4 was "yes", we obtain that

$$
0<1+a b+a c+a d+b c+b d+c d+a b c d
$$

Turning to the elementary solution to Problem 19 again, the inequality mentioned there is equivalent to the inequalities

$-1-a b-a c-a d-b c-b d-c d-a b c d<a+b+c+d+a b c+a b d+a c d+b c d$

and

$a+b+c+d+a b c+a b d+a c d+b c d<1+a b+a c+a d+b c+b d+c d+a b c d$ 


$$
\text { "tmcs-szalay" — 2010/4/12 — 23:36 — page } 71 \text { — \#11 }
$$

Now the first inequality is true, because

$$
-(1+a)(1+b)(1+c)(1+d)<0
$$

and the second inequality is true because

$$
0<(1-a)(1-b)(1-c)(1-d) .
$$

Each inequality with four variables is a source of inequalities with three variables. (Similarly to Problem 4.2.)

\section{Other special problems}

Each problem is a source of further problems. For example, by Problem 1 we construct some new problems. Having that $b \in \underline{\mathbb{R}} \Rightarrow(-b) \in \underline{\mathbb{R}}$, Problem 1 yields

Problem 20. Is it true that if $-1<a, b<1$ then

$$
-1<\frac{a-b}{1-a b}<1 \text { ? (Answer: "yes".) }
$$

Having that $1<a, b \Rightarrow \frac{1}{a}, \frac{1}{b} \in(0,1)$, Problem 1 yields

Problem 21. Is it true that if $1<a, b$ then

$$
0<\frac{a+b}{1+a b}<1 \text { ? (Answer: "yes".) }
$$

For any $\alpha, \beta \in \mathbb{R} \Rightarrow \sin \alpha, \cos \beta \in[-1,1]$. Problem 1 yields

Problem 22. Solve the inequality

$$
-1<\frac{\sin \alpha+\cos \beta}{1+\sin \alpha \cdot \cos \beta}<1 \text { ! }
$$

(Solution: $\alpha, \beta \in \mathbb{R}$ such that $\alpha \neq(2 n+1) \frac{\pi}{2}, n \in \mathbb{N}$ or $\beta \neq m \pi, m \in \mathbb{N}$.) Of course, we can find a more difficult problem, too.

Problem 23.

$$
\begin{gathered}
\max _{(x, y) \in \mathbb{R}^{2}} \frac{x^{2} y+x y^{2}+x+y}{x^{2} y^{2}+x^{2}+y^{2}+4 x y+1}=?, \\
\min _{(x, y) \in \mathbb{R}^{2}} \frac{x^{2} y+x y^{2}+x+y}{x^{2} y^{2}+x^{2}+y^{2}+4 x y+1}=?
\end{gathered}
$$


Solution to Problem 23. Assuming that point $(x, y) \neq(-1,1)$ or $(1,-1)$ we can write

$$
\begin{aligned}
& \frac{x^{2} y+x y^{2}+x+y}{x^{2} y^{2}+x^{2}+y^{2}+4 x y+1}=\frac{x\left(1+y^{2}\right)+y\left(1+x^{2}\right)}{\left(1+x^{2}\right)\left(1+y^{2}\right)+4 x y}= \\
& =\frac{\frac{x}{1+x^{2}}+\frac{y}{1+y^{2}}}{1+\frac{4 x y}{\left(1+x^{2}\right)\left(1+y^{2}\right)}}=\frac{1}{2} \frac{\frac{2 x}{1+x^{2}}+\frac{2 y}{1+y^{2}}}{1+\frac{2 x}{1+x^{2}} \frac{2 y}{1+x^{2}}} .
\end{aligned}
$$

Denoting $a=\frac{2 x}{1+x^{2}}, b=\frac{2 y}{1+y^{2}}$, we have

$$
\frac{x^{2} y+x y^{2}+x+y}{x^{2} y^{2}+x^{2}+y^{2}+4 x y+1}=\frac{1}{2} \frac{a+b}{1+a b} \text {. }
$$

Clearly, $0 \leq|a|,|b| \leq 1$. By the elementary solution to Problem 1 we have that if $|a|<1$ and $|b|<1$ then

$$
-\frac{1}{2}<\frac{x^{2} y+x y^{2}+x+y}{x^{2} y^{2}+x^{2}+y^{2}+4 x y+1}<\frac{1}{2} .
$$

On the other hand, if $x=1$ and $y \neq-1$ then $a=1$ and $b \neq-1$, (or if $x \neq-1$ and $y=1$ then $a \neq-1$ and $b=1$ ), so

$$
\frac{x^{2} y+x y^{2}+x+y}{x^{2} y^{2}+x^{2}+y^{2}+4 x y+1}=\frac{1}{2} .
$$

Moreover, if $x \neq-1$ and $y=-1$ then $a \neq 1$ and $b=-1$, (or if $x=-1$ and $y \neq 1$ then $a=-1$ and $b \neq 1$ ), so

$$
\frac{x^{2} y+x y^{2}+x+y}{x^{2} y^{2}+x^{2}+y^{2}+4 x y+1}=-\frac{1}{2} \text {. }
$$

So,

$$
\begin{aligned}
& \max _{\substack{(x, y) \in \mathbb{R}^{2} \\
(x, y) \neq(1,-1) \wedge(x, y) \neq(-1,1)}} \frac{x^{2} y+x y^{2}+x+y}{x^{2} y^{2}+x^{2}+y^{2}+4 x y+1}=\frac{1}{2}, \\
& \min _{\substack{(x, y) \in \mathbb{R}^{2} \\
(x, y) \neq(1,-1) \wedge(x, y) \neq(-1,1)}} \frac{x^{2} y+x y^{2}+x+y}{x^{2} y^{2}+x^{2}+y^{2}+4 x y+1}=-\frac{1}{2} .
\end{aligned}
$$

ISTVÁN SZALAY

UNIVERSITY OF SZEGED

HUNGARY

E-mail: szalay@jgytf.u-szeged.hu

(Received March, 2009) 